\title{
Analysis of the LQR algorithm in the field of testing the dynamics of quadcopter movement
}

\author{
LUCJAN SETLAK, RAFAŁ KOWALIK \\ Aviation Division, Department of Avionics and Control Systems \\ Polish Air Force University \\ Deblin 08-521, ul. Dywizjonu 303 No. 35 \\ POLAND \\ 1.setlak@law.mil.pl,r.kowalik@law.mil.pl
}

\begin{abstract}
The subject of this article is to present the issues related to the LQR control algorithm used in the linear model describing the dynamics of the flying object in terms of tracking its flight trajectory. The use of the LQR regulator is also a method of calculating the optimal $K$-feedback reinforcement, with this type of reinforcement used to control the system in the form of a control signal can be determined by tuning the $Q$ and $R$ weight matrix elements in the LQR method. Based on the above, the main research goal of the article is to develop an algorithm for the control system implemented on the quadrotor using the LQR method to obtain the best K-feedback gain in flight state with unstable motion. To this end, a mathematical model describing the essence of linear-quadratic control using the LQR controller is presented in this paper. It should be noted that due to the fact that only four states can be controlled at the same time in a quadrotor, hence the flight trajectories are determined on the basis of four states, while the three-dimensional position, position of the tested object in the coordinate system and rotation along the axis are described as deviation movement. In addition, the work also designed on the basis of the created linear model of a linear quadrotor LQR control approach for this model due to the fact that the performance of the linear model and non-linear model around a specified nominal point is almost identical. The control system based on the LQR algorithm was developed in the Matlab/Simulink environment, and the results obtained in the form of graphs for the quantities characterizing the dynamics of the tested object were used to assess the effectiveness of the LQR method used. In the final part of the work, practical conclusions have been formulated based on the research (analysis, models, simulations) and analysis of the results obtained.
\end{abstract}

Key-Words: - Analysis, linear quadratic regulator, testing, dynamics of movement, quadcopter

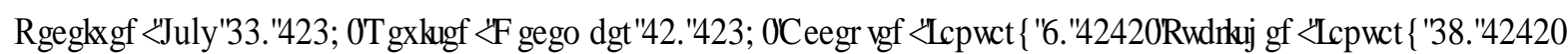

\section{Introduction}

One of the key elements of the unmanned aerial vehicle (UAV) design process is its comprehensive mass and balance analysis. The term "mass and balance" refers to the mass properties of the aircraft (object) and the resulting stability or instability.

In turn, the term "mass properties" usually refers to the following quantities: mass, center of gravity (center of mass), moment of inertia. The center of gravity is the point where the sum of moments derived from the mass of the object equals zero.

Therefore, this point is the point relative to which the aircraft is balanced and at which mass and gravitational forces are attached. For the needs of the work, each element of the aircraft was weighed and the center of gravity of each was determined.

The center of gravity of the entire aircraft was determined using a simple geometric sum and parallel axis theorem [1], [2].

The mass of individual aircraft components is shown in the table below (Table 1).
Table 1 Mass of individual elements

\begin{tabular}{|c|c|}
\hline Element & Mass [g] \\
\hline Propeller & 100 \\
\hline Radio control electronics & 65 \\
\hline Camera & 55 \\
\hline Batteries & 350 \\
\hline Wires & 29 \\
\hline Fuselage & 750 \\
\hline Sum & $\mathbf{1 3 4 9}$ \\
\hline
\end{tabular}

The load capacity (load) of the aircraft was placed in such a way as to shift the center of gravity to the desired position in front of the aerodynamic center [3], [4].

The exact position of the center of gravity should be made when trimming the aircraft to obtain longitudinal stability.

The equations of the moments of inertia are as follows (1) - (5) [5], [6]: 


$$
\begin{gathered}
I_{y y}=\sum_{i=1}^{n} m i\left\{\left(Z_{i}-Z_{c g}\right)^{2}+\left(X_{i}-X_{c g}\right)^{2}\right\} \\
I_{z z}=\sum_{i=1}^{n} m i\left\{\left(X_{i}-X_{c g}\right)^{2}+\left(Y_{i}-Y_{c g}\right)^{2}\right\} \\
I_{x y}=\sum_{i=1}^{n} m i\left\{\left(X_{i}-X_{c g}\right)^{2}\left(Y_{i}-Y_{c g}\right)^{2}\right\}=0 \\
I_{y z}=\sum_{i=1}^{n} m i\left\{\left(Y_{i}-Y_{c g}\right)^{2}\left(Z_{i}-Z_{c g}\right)^{2}\right\}=0 \\
I_{z x}=\sum_{i=1}^{n} m i\left\{\left(Z_{i}-Z_{c g}\right)^{2}+\left(X_{i}-X_{c g}\right)^{2}\right\}
\end{gathered}
$$

It should be noted that the design of the flying wing does not distinguish between wings and the fuselage and there is no vertical and horizontal stabilizer. Due to this, this type of solution not only increases the lift to resistance ratio, reducing the effective reflection surface, but also increases the range of the flight and reduces energy consumption.

However, this type of construction caused many problems in the design of the flight control system. On the one hand, due to the shape of the aircraft (large ratio of width to length) and the lack of a horizontal stabilizer, which reduces longitudinal static stability and control efficiency.

On the other hand, the lack of vertical fin causes a decrease in transverse lateral attenuation. In this case, a new control mechanism should be attached to the structure to allow rotation of the aircraft around the vertical axis.

The LQR (Linear Quadratic Regulator) linearquadratic regulator technology is widely used in many engineering fields, especially in the design of unmanned aerial vehicle flight control systems using feedback [7], [8], [9].

This method has many advantages, such as: a phase margin greater than $60^{\circ}$, an infinite margin of amplitude and high reliability.

The use of LQR technology has allowed to increase the flight stability of unmanned aircraft in a classic system, which solves the problem of vulnerability associated with air blasts.

Currently, flying wing control systems mainly use classical control theory based on the use of square root method and frequency band analysis method.

It should be noted that despite the reliability and intuitiveness of this type of approach, a lot of work should be done to design a feedback loop using classic control methods, and sometimes it is difficult to meet the design requirements of a complex flight control system with multiple inputs and outputs and a strong connection.

LQR technology is most often used in UAV control systems in the classic system and unmanned helicopters [10], [11], [12].

This paper presents the use of the LQR method in the UAV control system - flying wing. This allowed not only to increase longitudinal static stability, but also to improve the dynamic characteristics of the object.

In addition, the amount of feedback has been reduced, which contributes to the use of this type of method, primarily for its convenience, in practical applications.

The controller has high reliability and a simple structure, implements control with zero static error of speed and angle of inclination.

The article uses the Matlab/Simulink environment to simulate the control method, while the results show that the longitudinal control based on LQR technology can make the UAV object design - flying wing achieve satisfactory longitudinal flight properties.

\section{Mathematical model of the flying wing in the scope of increasing longitudinal stability}

Due to the fact that the fuselage of the flying wing is short, the efficiency of controlling the rudder and elevons is low. In order to increase maneuverability, static stability must therefore be reduced accordingly.

In the event that the aircraft moves at subsonic speed or at large angles of attack, the aerodynamic focus moves backwards, reducing longitudinal static stability.

As a compromise between longitudinal stability and maneuverability, the center of gravity of the aircraft can be located between the aerodynamic focus at high angles of attack and high speeds and the aerodynamic focus at low angles of attack and low speeds.

Therefore, static stability can be maintained at low speeds and low angles of attack, while instability at high speeds and high angles of attack is still acceptable.

In view of the above reasoning, only at high speeds and a large angle of attack it is needed to strengthen longitudinal stability. 


\subsection{Feedback system (output)}

Despite the fact that by using a traditional linearquadratic regulator, it is possible to achieve an increase in aircraft stability, however, information on all system state variables and large workloads are necessary, which is not conducive to using this method in practical applications [13], [14], [15].

In connection with the above, a feedback system (output) was used.

For the needs of mathematical analysis, the following three rudders models were adopted:

Elevator: $\delta_{e}=\frac{20}{S+20} u_{e}$, engine control lever: $\delta_{t}=\frac{20}{s+20} u_{t}$, rudder at the wing tips: $\delta_{d}=\frac{40}{s+20} u_{d}$.

The enhanced state equation and the output equation can be represented in the following form (6):

$$
\left\{\begin{array}{c}
\dot{x}=A x+B u \\
y=C x
\end{array}\right.
$$

where: $x=\left[\Delta v \Delta \alpha \Delta q \Delta \theta \Delta \delta_{e} \Delta \delta_{t} \Delta \delta_{d}\right]^{T}$ - is the system state vector, $u=\left[\Delta u_{e} \Delta u_{t} \Delta u_{d}\right]^{T}$ - is the control vector, while $y=[\Delta v \Delta \alpha \Delta q \Delta \theta]^{T}$ - is the output signal.

The output form takes the form (7):

$$
u=K y
$$

where: $K$ - is a returnable profit matrix with an appropriate dimension.

After substituting equation (7) for equation (6), the following closed loop system equation (8) was obtained:

$$
x=(A+B \dot{K} C) x=A^{*} x u=K y
$$

The purpose of developing the principle of stability enhancement control is to adjust the state of the aircraft in such a way that the initial conditions errors are kept at zero, which can ensure the stability of the flight of the object.

Thus, the following function can be minimized by selecting the control input signal $u$ (9):

$$
J=\frac{1}{2} \int_{0}^{\infty}\left(x^{T} Q x+u^{T} R u\right) d t
$$

where: $Q$ - is a semi-positive symmetrical matrix and $R$ - a positive symmetrical matrix.

After substituting equation (7) and (6) with the above equation (9), we obtained (10):

$$
J=\frac{1}{2} \int_{0}^{\infty} x^{T}\left(Q+C^{T} K^{T} R K C\right) x d t
$$

It can be stated that the selection of an appropriate $K$-feedback matrix will minimize the function (10), which changes the problem of dynamic optimization into an easily soluble static problem.

It has been assumed that a positively defined symmetric matrix $P$ can be determined to build the Lapunov $x$ function [16], [17], [18].

If the function satisfies Lapunov's theorem on stability, the closed-loop system (8) is asymptotically stable.

The Lapunov's function can be defined as (11):

$$
V(x)=x^{T} P x
$$

Using the formula (8), the derivative $V(x)$ expressed in the form (12) was obtained:

$$
\dot{V}(x)=x^{T}\left(A^{* T} P+P A^{*}\right)
$$

Then, after integrating the function (10) and using the properties of the Lapunov $V(x)$ function, the following equation (13) was obtained:

$$
\begin{aligned}
\frac{d\left(x^{T} P x\right)}{d t} & =x^{T}\left(A^{* T} P+P A^{*}\right) x \\
& =-x^{T}\left(Q+C^{T} K^{T} R K C\right) x
\end{aligned}
$$

In turn, since it was assumed that the closed-loop system is asymptotically stable, function (10) can be written as (14):

$$
\begin{gathered}
J=\frac{1}{2} x^{T}(0) P x(0)-\lim _{t \rightarrow \infty} \frac{1}{2} x^{T}(t) P x(t) \\
=\frac{1}{2} x^{T}(0) P x(0)
\end{gathered}
$$

From equation (14) the closed loop system function can be calculated provided that the initial state $x(0)$ is known.

Because equation (13) must meet all initial conditions, all trajectories of state $x(0)$ satisfy the following Lapunov equation (15):

$$
f=Q+C^{T} K^{T} R K C+A^{* T} P+P A^{*}=0
$$

From equation (15) it can be stated that if $Q$ and $K$ matrices are given, the auxiliary matrix $P$ can be determined by the Lapunov function and is independent of the state of the system.

To sum up, when it comes to any $K$-feedback matrix with a fixed value, if there is a nonnegatively defined symmetric matrix $P$ that satisfies the Lapunov equation (15) and the closed-loop system is stable, the quadratic efficiency factor is significant for the initial condition $x(0)$ and matrix $P$, independent of system states [19], [20]. 
To simplify the feedback solution $K$, the relationship $\operatorname{tr}(\mathrm{AB})=\operatorname{tr}(\mathrm{BA})$ was introduced, which describes the relationship of matrix traces.

Therefore, equation (14) can be presented as follows (16):

$$
J=\frac{1}{2} \operatorname{tr}\left[\operatorname{Px}(0) x^{T}(0)\right]
$$

Under conditions of limiting the equations of state (8), the problem that was obtained after minimizing the function (10) is transformed into a problem that solves the feedback matrix $K$ by minimizing the equation (16) using equation (15) with the auxiliary symmetric matrix $P$.

However, occurring in equation (16), $x(0) x^{T}(0)$ depend on the initial conditions that engineering is not known in many practical aspects.

Therefore, it was assumed that the initial state $x(0) x^{T}(0)$ is a unit matrix.

In this case, the problem of solving performance indicators (16) is transformed in solving the expectations of performance indicators $E(J)$, which eliminates initial conditions.

Then, the solution of the $K$ matrix and the $P$ matrix was started. To this end, the Lagrange matrix factor $\lambda \in R^{3 \times 4}$ was introduced and the Hamilton function (17) was written:

$$
H=F+\operatorname{tr}(f \lambda)
$$

where: $F=\operatorname{tr}\left[P x(0) x^{T}(0)\right]$.

Then differentiation (17) was made after $K, P, \lambda$ respectively, and the results were compared to zero (18):

$$
\left\{\begin{array}{c}
\frac{\partial H}{\partial K}=R K C \lambda C^{T}-B^{T} P \lambda C^{T}=0 \\
\frac{\partial H}{\partial P}=A^{*} \lambda+\lambda A^{* T}+x(0) x^{T}(0)=0 \\
\frac{\partial H}{\partial \lambda}=Q+C^{T} K^{T} R K C+A^{* T} P+P A^{*}=0
\end{array}\right.
$$

The above three equations are the necessary conditions for feedback, where $R$ is a positively determined asymmetrical matrix, so the $K$-feedback matrix can be defined as (19) [21], [22], [23]:

$$
K=R^{-1} B^{T} P \lambda C^{T}\left(C \lambda C^{T}\right)^{-1}
$$

As a result, the formula for strengthening the longitudinal stability of the flying wing was obtained (20):

$$
u=-K y=-R^{-1} B^{T} P \lambda C^{T}\left(C \lambda C^{T}\right)^{-1} C x
$$

A schematic diagram of the longitudinal stability strengthening system, designed with the help of feedback, is presented above (Fig. 1).

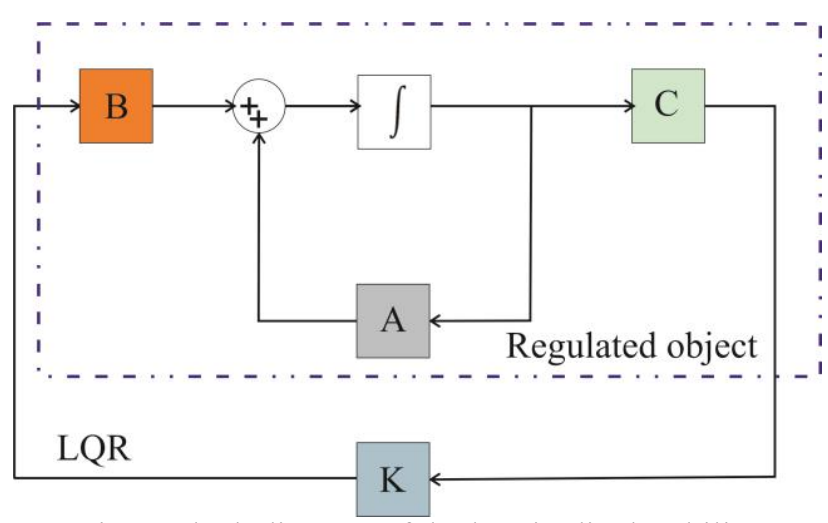

Fig. 1 Block diagram of the longitudinal stability strengthening system

The iterative method was used to solve the $K$-feedback matrix. Below are the individual stages (steps) of the method used:

Step 1: Initialization of parameters

$i=0$ is taken, and then an asymptotically stable matrix $A_{i}=A-B K_{i} C$ is created, selecting the initial reinforcement $K_{i}$.

Step 2: Iteration process

I-th iteration is performed and $P_{i}, \lambda_{i}$ and function $J_{i}=\frac{1}{2} \operatorname{tr}\left[P_{i} x(0) x^{T}(0)\right]$ are solved using the following Lapunov equations (21):

$$
\left\{\begin{array}{c}
Q+C^{T} K_{i}^{T} R K_{i} C+A_{i}^{* T} P+P A_{i}^{*}=0 \\
A_{i}^{*} \lambda_{i}+\lambda_{i} A_{i}^{* T}+x(0) x^{T}(0)=0
\end{array}\right.
$$

The correction of the value of the feedback matrix $K$ was calculated based on the formula $\Delta \mathrm{K}=R^{-1} B^{T} P_{i} \lambda_{i} C^{T}\left(C \lambda_{i} C^{T}\right)^{-1}-K_{i}$ and the changed matrix has the value $K_{i+1}=K_{i}+\varepsilon \Delta K$.

Then the $\varepsilon$ value was chosen so that the matrix $A_{i+1}$ would be asymptotically stable while searching for the value $J_{i+1} \leq J_{i}$. In case $J_{i+1}$ is close to $J_{i}$ you can proceed to step three. Otherwise, $i=i+1$ is set and returns to step two, continuing the calculation.

Step 3: Estimation

$\mathrm{K}=K=K_{i}$ and $J=J_{i}$ are determined.

\section{Selected simulation tests on the example of a single wing flight dynamics simulation}

The following parameters were adopted for the simulation: height $\mathrm{h}=2000 \mathrm{~m}$ and flight speed $\mathrm{Ma}=0.805$. The equation of state and the output equation take the following form (22) - (24):

$$
\left\{\begin{array}{c}
\dot{x}_{m}=A_{m} x_{m}+B_{m} u \\
y=C_{m} x_{m}+D_{m} u
\end{array}\right.
$$

where:

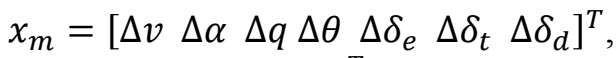

$u_{m}=\left[\begin{array}{lll}\Delta u_{e} \Delta u_{t} \Delta u_{d}\end{array}\right]^{T}$. 


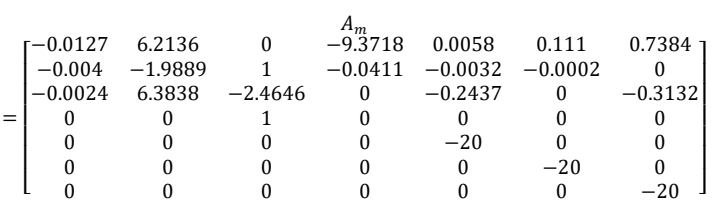

$A_{m} B_{m}=\left[\begin{array}{ccc}0 & 0 & 0 \\ 0 & 0 & 0 \\ 0 & 0 & 0 \\ 0 & 0 & 0 \\ 20 & 0 & 0 \\ 0 & 20 & 0 \\ 0 & 0 & 40\end{array}\right] \quad C_{m}=\left[\begin{array}{ccccccc}1 & 0 & 0 & 0 & 0 & 0 & 0 \\ 0 & 57.3 & 0 & 0 & 0 & 0 & 0 \\ 0 & 0 & 57.3 & 0 & 0 & 0 & 0 \\ 0 & 0 & 0 & 57.3 & 0 & 0 & 0\end{array}\right]$
$D_{m}=\left[\begin{array}{ccc}0 & 0 & 0 \\ 0 & 0 & 0 \\ 0 & 0 & 0 \\ 0 & 0 & 0\end{array}\right]\left\{\begin{array}{l}\dot{x}_{m}=A_{m} x_{m}+B_{m} u \\ y=C_{m} x_{m}+D_{m} u\end{array}\right.$

The eigenvalues of the open system according to formula (36) are: $\lambda_{1}=-4.5775, \lambda_{2}=0.4419$, $\lambda_{3,4}=-0.0665 \pm 0.359 i, \lambda_{5,6,7}=-20$,

where: $\lambda_{1,2}$ - define the appropriate elements characteristic of the short period, and $\lambda_{3,4}$ - are the appropriate characteristic elements of the long period.

The above eigenvalues play a decisive role in the longitudinal movement of the aircraft [24], [25], [26], [27]. In the event that the aircraft is subjected to external interference or a given input signal, the rule of changing longitudinal parameters over time is the superposition of two moves, with $\lambda_{5,6,7}$ being loops of three rudders.

System responses to the $1^{\circ}$ the angle of attack disruption are illustrated by subsequent drawings (Figs. 2-5).

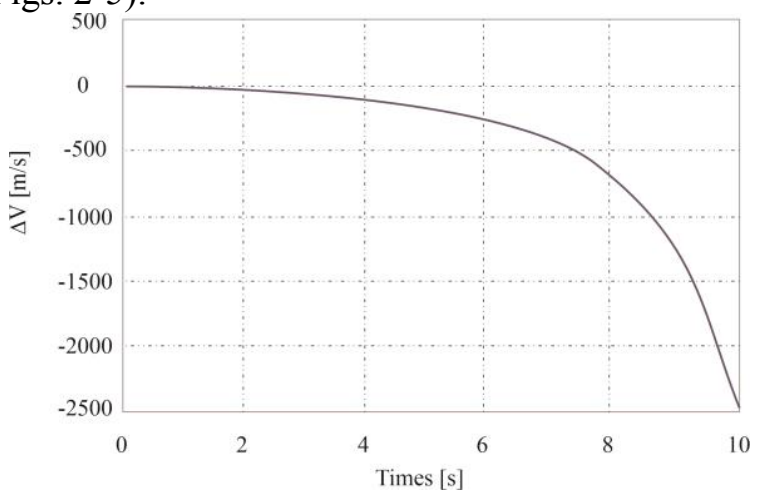

Fig. 2 Flight speed response curve

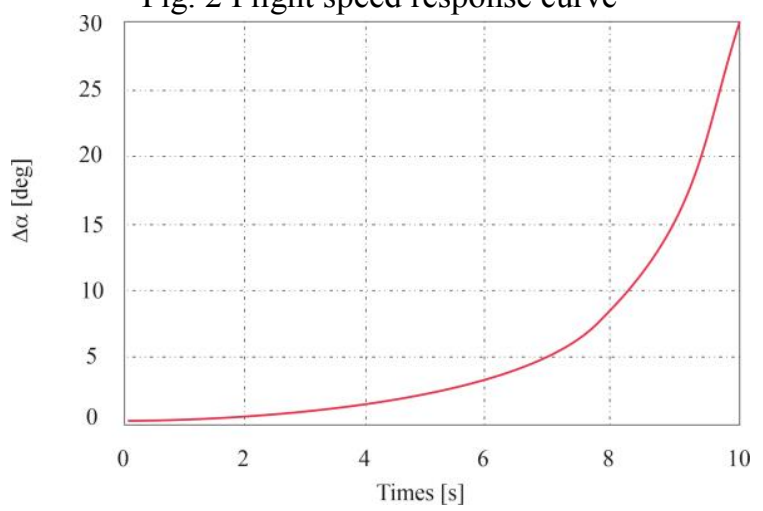

Fig. 3 Angle of attack response curve

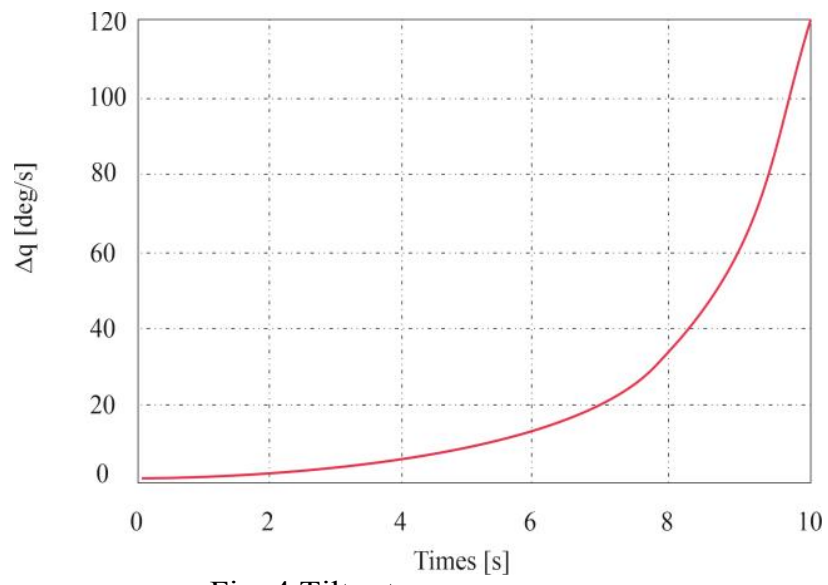

Fig. 4 Tilt rate response curve

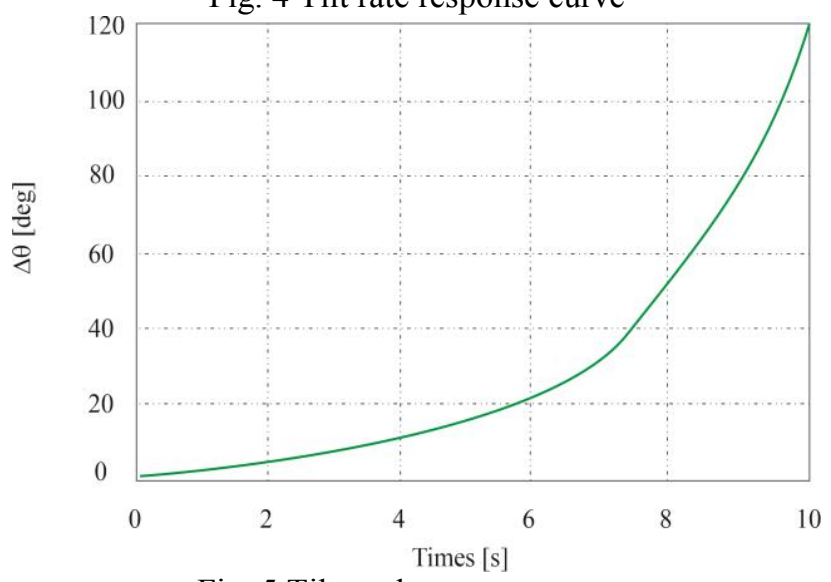

Fig. 5 Tilt angle response curve

The above drawings show that the disorder is getting worse. That is why it is important to introduce a longitudinal stability strengthening rule.

The rule for strengthening the longitudinal stability of an aircraft can be determined as $u=K y$ by applying the LQR method, where $K \in R^{3 \times 4}$.

To achieve a satisfactory stability enhancing effect, appropriate $Q$ and $R$ matrices should be selected before applying LQR technology [28], [29], [30], [31].

In turn, taking into account the fact that longitudinal static instability causes disturbance in the short term, in the function of cost, the state $\Delta \alpha^{2}$ and $\Delta q^{2}$ should be weighted by the element $q_{a}$ of the matrix $Q$.

In the long-term mode, with suppression based on the characteristic values of $\lambda_{3,4}$, it can be seen that the state $\Delta v^{2}$ i $\Delta \theta^{2}$ must be weighted by the element $q_{b}$ of the matrix $Q$. Since extended state variables are not discussed, it is not necessary to give them weight.

As a result, the weighted matrix $Q$ can be written as $Q=\operatorname{diag}\left\{q_{b}, q_{a}, q_{a}, q_{b}, 0,0,0\right\}$. However, when it comes to the $R$ matrix, the form $R=\rho \times I$ is used to prevent excessive input control, where $\rho$ is the design parameter and $I$ is the unit matrix with the corresponding dimension. 
After repeated selection and checking, it was found that the longitudinal stability reinforcement will achieve the best result, when $Q=\operatorname{diag}\{50,10,10,50,0,0,0,0\}$, and $\rho=1$.

The optimal $K$-feedback matrix was obtained using the iterative method described in the paper as described in the expression (25). The change of the cost function during the iteration process is presented in the figure below (Fig. 6).

$=\left[\begin{array}{cccc}-1.6073 & -22.8329 & -23.3958 & -26.5004 \\ 7.2136 & 10.1877 & 0.9967 & -14.1970 \\ 3.2225 & 2.4844 & -2.4463 & -7.6770\end{array}\right]$

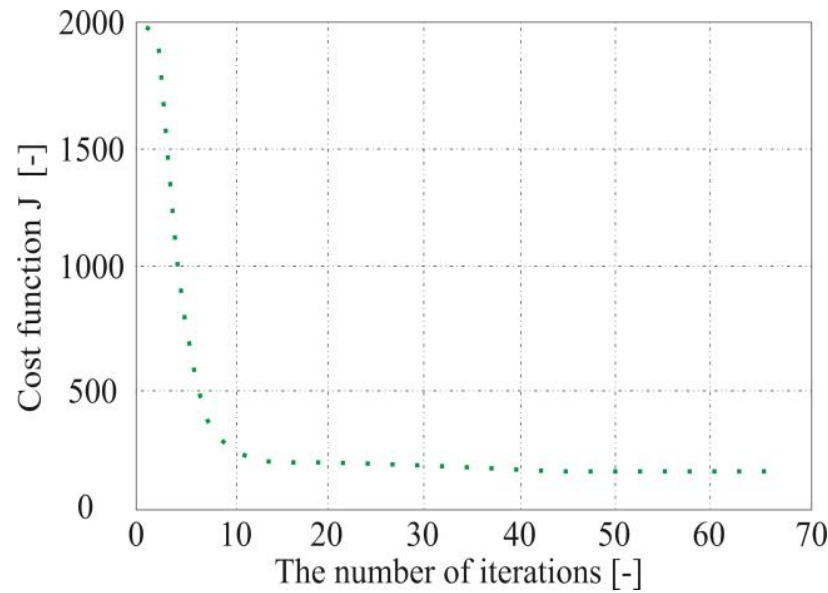

Fig. 6 Change in the curve of the cost function $\mathrm{J}$

The eigenvalues of the closed system after strengthening the stability are as follows: $\lambda_{1,2}=-1.526 \pm 0.764 i, \quad \lambda_{3,4}=-10.437 \pm 9.01 i$, $\lambda_{5}=-5.884, \lambda_{6}=-14.657, \lambda_{7}=-20$.

The responses of the closed system to a $1^{\circ}$ the angle of attack are illustrated in the following figures (Figs. 7-10).

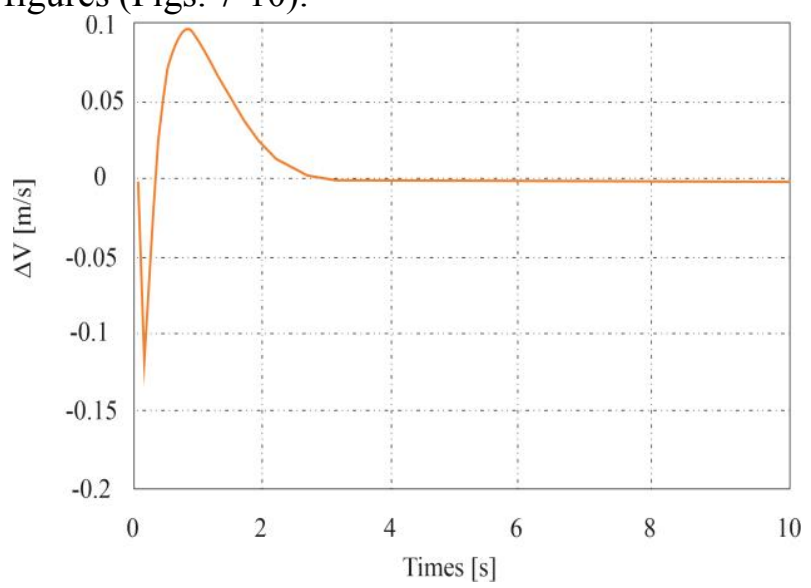

Fig. 7 Flight speed response curve

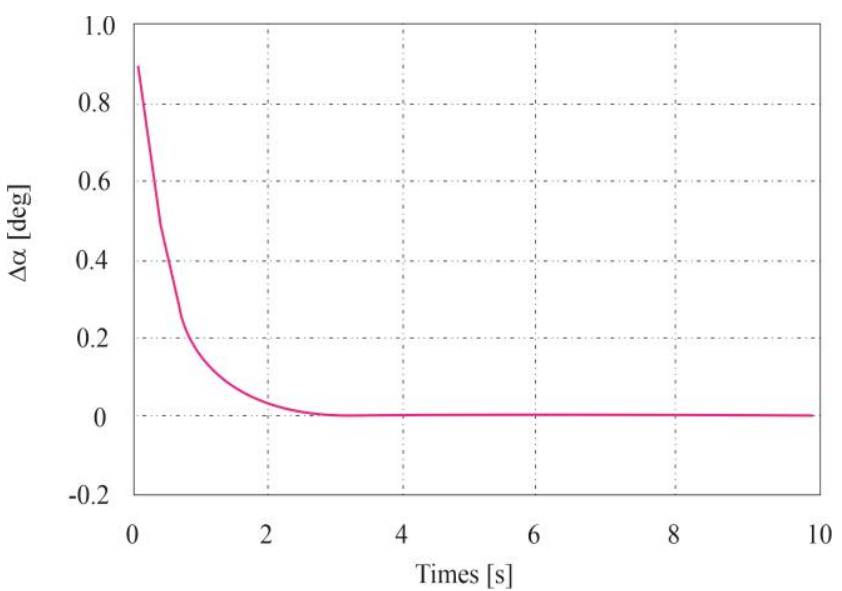

Fig. 8 Angle of attack response curve

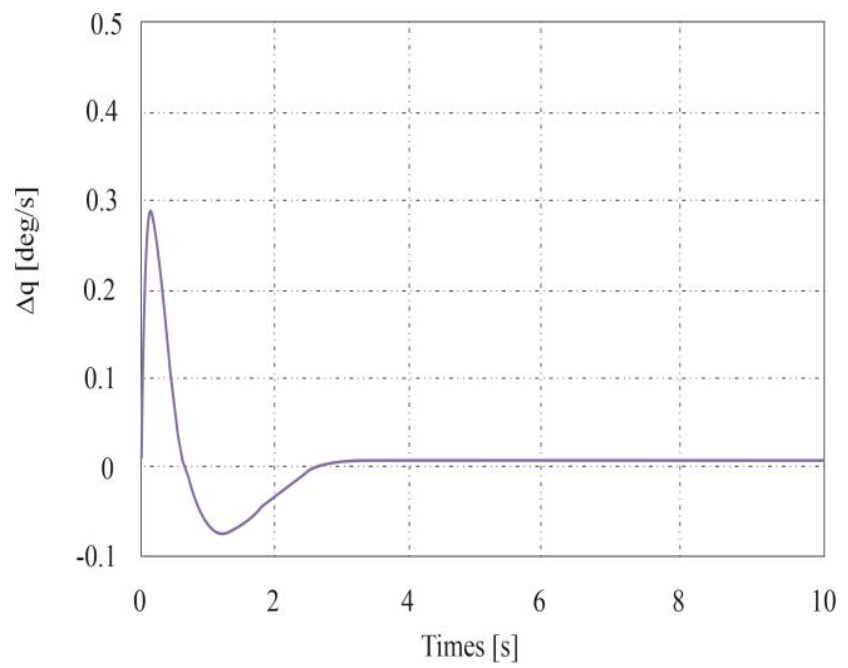

Fig. 9 Tilt rate response curve

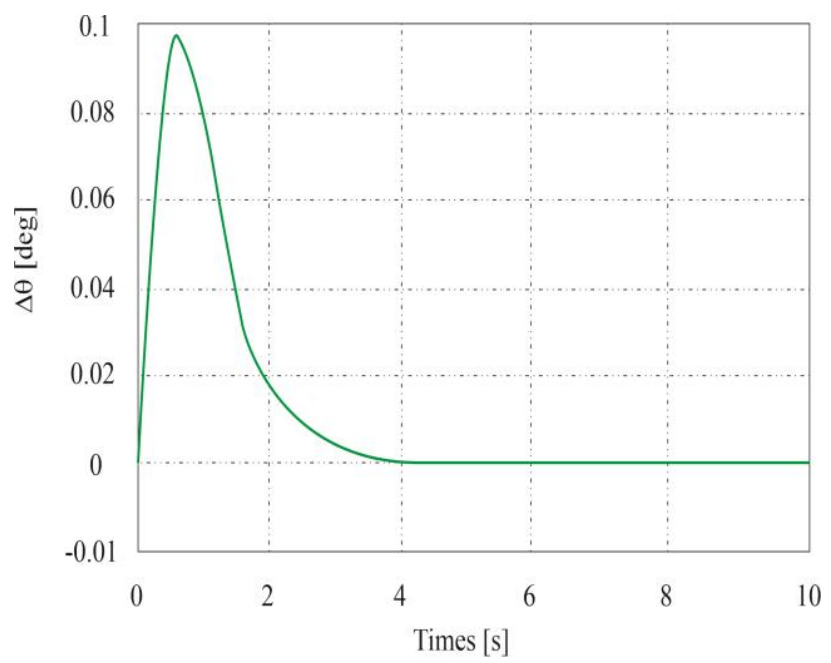

Fig. 10 Tilt angle response curve

After achieving stability reinforcement, position control was based on it in the longitudinal plane. To check the two different position control methods, only flight speed and tilt angle were controlled.

Parameters $\Delta v$ and $\Delta \theta$ were selected as the system output, while $u_{t}$ and $u_{e}$ - as the control input signal. 
The LQR methods described in the previous chapter were used to develop the position control rule $u_{t}$ and $u_{e} \mathrm{w}$ ten sposób, aby możliwe było osiągnięcie śledzenia poleceń prędkości lotu i kąta pochylenia. It was assumed that all system states are measurable except the angle of attack, which can be estimated by the reducing system.

W oparciu o powyższe, równanie stanu oraz równanie wyjściowe po wzmocnieniu stateczności zapisać można jako (26):

where:

$$
\left\{\begin{array}{c}
\dot{x}(t)=A^{*} x(t)+B^{*} u(t) \\
y(t)=C^{*} x(t)
\end{array}\right.
$$

$x=\left[\Delta v \Delta \alpha \Delta q \Delta \theta \Delta \delta_{e} \Delta \delta_{t}\right]^{T}$,

$u=\left[\Delta u_{t} \Delta u_{e}\right]^{T}, y=[\Delta v \Delta \theta]^{T}$.

Referring to the LQR method supported by command tracking, the system of equations (26) can receive a new reinforced state equation in the form (27) [32], [33], [34]:

$$
\dot{\tilde{x}}=\tilde{A} \tilde{x}+\tilde{B} \tilde{u}
$$

After repeated selection and checking, it was found that command tracking would achieve the best results when

$R=\operatorname{diag}\{1,1\}$,

$Q=\operatorname{diag}\{20,20,20,20,1,1,500,2000\}$.

The optimal feedback matrix $K$ was obtained as follows (28):

$K=\left[\begin{array}{llllllll}k_{\Delta \dot{v}}^{u_{t}} & k_{\Delta \dot{u}}^{u_{t}} & k_{\Delta \dot{q}}^{u_{t}} & k_{\Delta \dot{\theta}}^{u_{t}} & k_{\Delta \delta_{e}}^{u_{t}} & k_{\Delta \delta_{t}}^{u_{t}} & k_{\Delta e_{v}}^{u_{t}} & k_{\Delta e_{\theta}}^{u_{u_{t}}} \\ k_{\Delta \dot{v}}^{u_{e}} & k_{\Delta \dot{e}}^{u_{e}} & k_{\Delta \dot{q}}^{u_{e}} & k_{\Delta e}^{u_{e}} & k_{\Delta \delta_{e}}^{u_{e}} & k_{\Delta \delta_{t}}^{u_{e}} & k_{\Delta e_{v}}^{u_{e}} & k_{\Delta e}^{u_{e}}\end{array}\right]$
$=\left[\begin{array}{ccccccccc}-14.59 & 90.15 & 90.17 & 380.32 & -4.77 & -0.012 & 7.89 & -185.56 \\ -27.22 & -62.95 & 8.57 & 116.62 & -0.018 & -3.62 & 11.75 & 124.75\end{array}\right]$

Finally, the rule for controlling the flight speed and inclination angle UAV - a flying wing, developed using the LQR method supported by command tracking, is as follows (29):

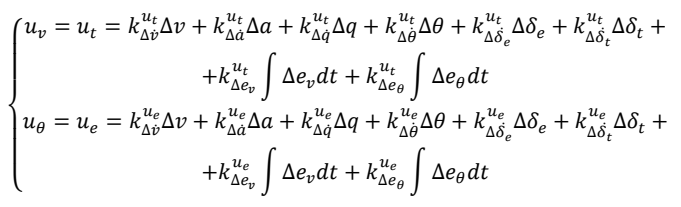

Using the LQR method supported by apparent command tracking and developing a system to reduce the state variable $\Delta \alpha$ that cannot be measured, the process is identical to the above.

The observer's output error error matrix takes the form $\bar{K}_{e}=\left[\begin{array}{lll}0.3615 & 0.3714 & 0\end{array}\right]$.

Finally, the rule for controlling the flight speed and angle of inclination UAV - a flying wing, developed using the LQR method supported by apparent command tracking, is as follows (30):

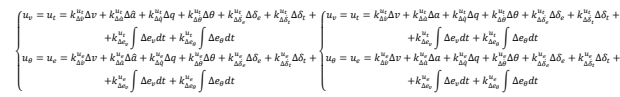

Both control rules were used to simulate the UAV model. Formula (29) was named "controller $1 "$ and formula (30) - "controller 2".

The following parameters were adopted: height $h=2000 \mathrm{~m}$ and flight speed $M a=0.805$, and also that the initial state of the system is equilibrium. At $t=1 \mathrm{~s}$, the flight speed changes by $50 \mathrm{~m} / \mathrm{s}$, and the angle of inclination by $5^{\circ}$. The simulation results are illustrated in the figures below (Figures 11-14).

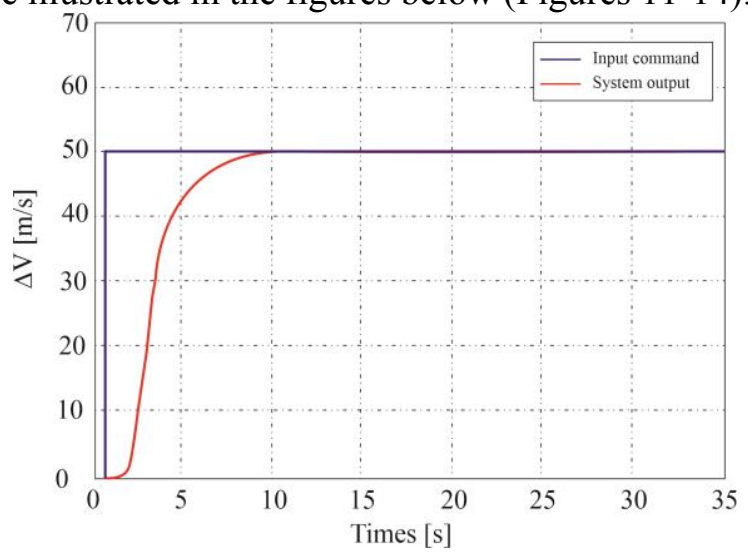

Fig. 11 Flight speed response curve (controller 1)

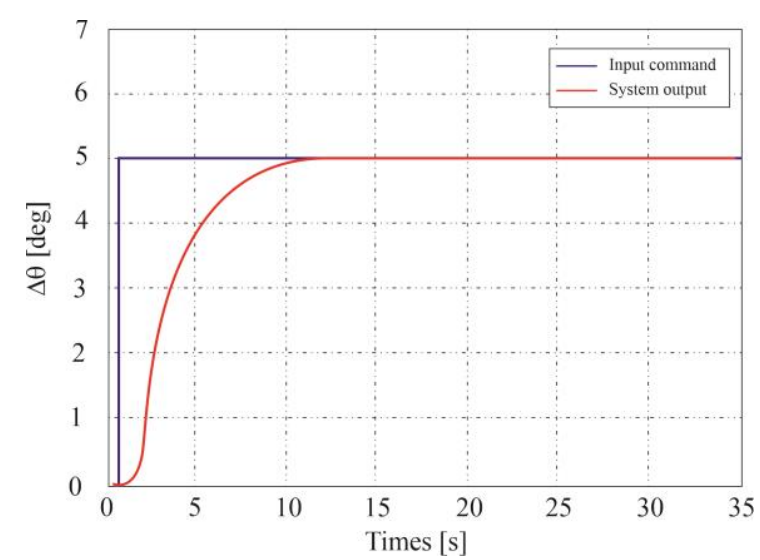

Fig. 12 Tilt angle response curve (controller 1)

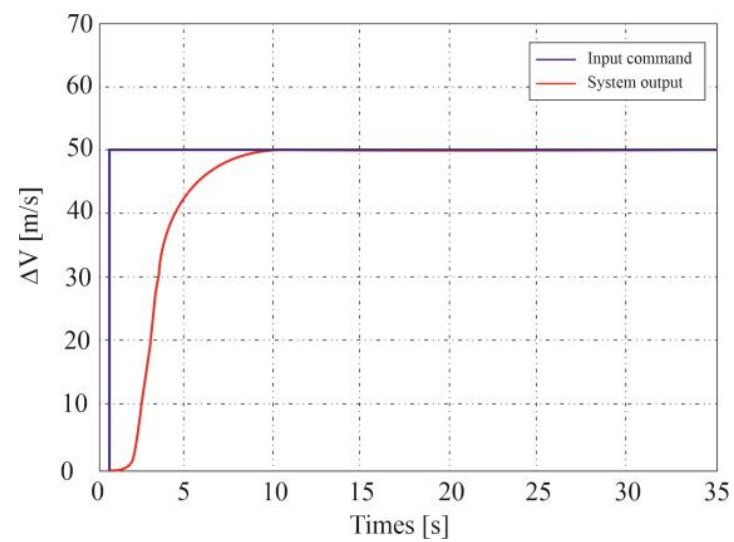

Fig. 13 Flight speed response curve (controller 2) 


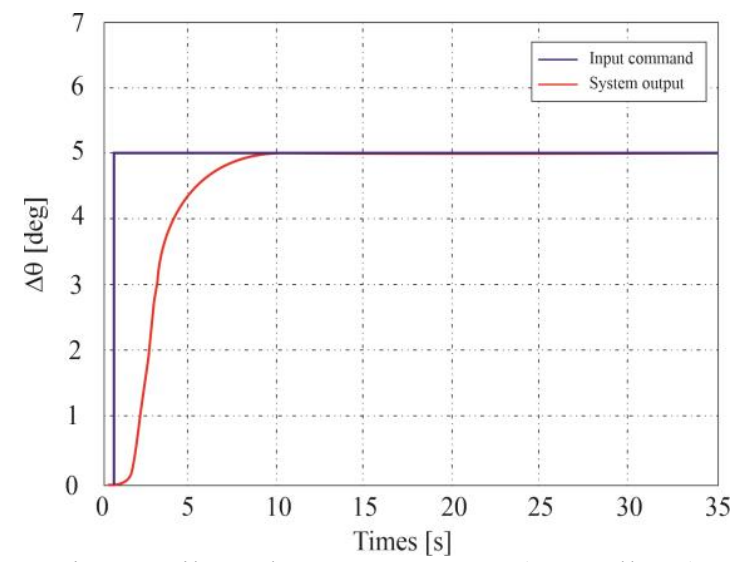

Fig. 14 Tilt angle response curve (controller 2)

The above figures show that both input signals of both controllers can be tracked without a fixed system error. In addition, there is high precision, short transition and small overshooting of the response process.

In addition, since the controller 2 has a reducing system to analyze the effect of following commands, a difference was made between the figures (Figs. 11-14), after which the output signal difference curves were obtained for two different methods illustrated in the following figures (Figs. 15-16).

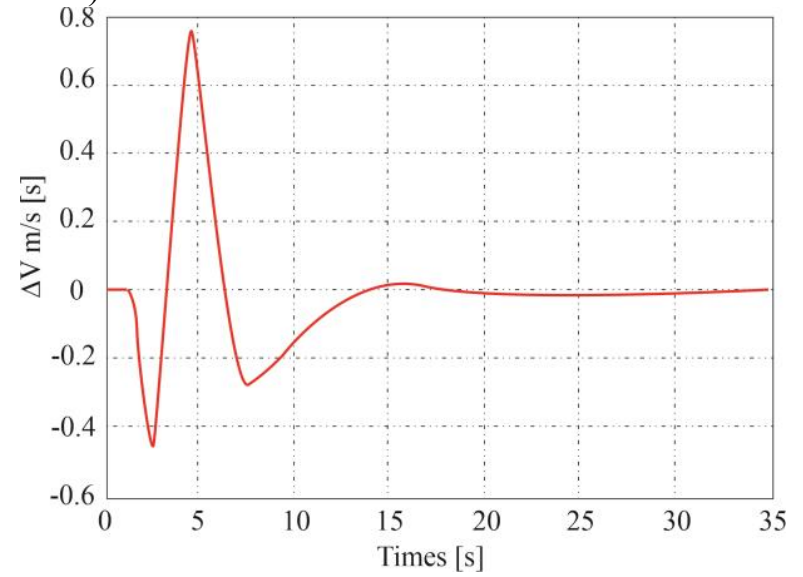

Fig. 15 Flight speed difference curve

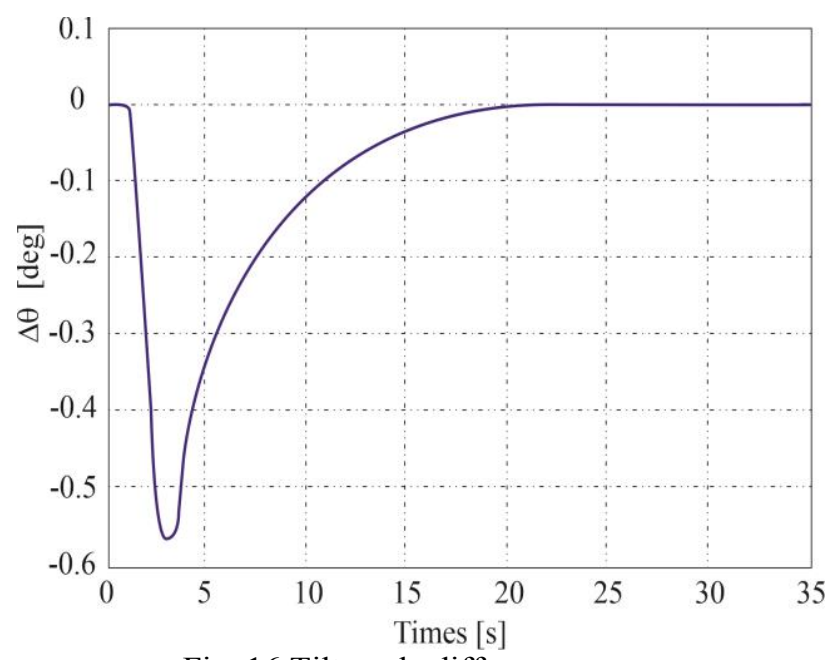

Fig. 16 Tilt angle difference curve
The below two figures confirm that the varying range of input signal difference curves is small throughout the entire response process.

It can therefore be concluded that the introduction of a reduction circuit has little effect on command tracking, and controller 2 maintains almost full performance of controller 1 .

\section{Conclusions}

The aircraft in the system controlled by the LQR algorithm is statically stable, but has a very high inclination moment factor.

In addition, the control surfaces in the form of a single wing of the mother aircraft are insufficient to ensure equilibrium for flight conditions just before the separation process.

These problems were tried to be solved by moving the object relative to the mother plane, but unfortunately no satisfactory results were achieved.

The aircraft in the classic configuration has sufficiently large control surfaces to ensure equilibrium for the conditions just before the separation process [35], [36].

In addition, the volume feature of the tail was changed to 0.298 , and the rocket plane was wedged in relation to the carrier in such a way that in flight conditions just before separation it was possible to achieve equilibrium without deflecting the control surfaces.

This wedge angle is -4.4 degrees.

\section{References:}

[1] M. Alhaddad, M. Tyupkin, and L. Aeshina, "Modeling and Attitude Control of a Quadcopter Using Linear Quadratic Regulator," 2016, pp. 173-176.

[2] R. Bonna and J. Camino, "Trajectory Tracking Control of a Quadrotor Using Feedback Linearization," in Proceedings. of the XVII International Symposium on Dynamic Problems of Mechanics DINAME-2015, 2015.

[3] J. P. Hespanha, "Linear Systems Theory," Princeton, NJ, USA: Princeton University Press, 2009.

[4] S. Bouabdallah, and R. Siegwart, "Backstepping and sliding-mode techniques applied to an indoor micro quadrotor," in Robotics and Automation, 2005. ICRA 2005. Proceedings of the 2005 IEEE International Conference, 2005, pp. 2247-2252.

[5] A. Chovancová, T. Fico, P. Hubinský, and F. Duchoň, "Comparison of various quaternionbased control methods applied to quadrotor 
with disturbance observer and position estimator," Robotics and Autonomous Systems, Vol. 79, 2016, pp. 87-98.

[6] L. Setlak, R. Kowalik, "Studies of 4-rotor unmanned aerial vehicle UAV in the field of control system," 22nd International Conference on Circuits, Systems, Communications and Computers, CSCC 2018, MATEC Web of Conferences, Vol. 210, 2018.

[7] A. Kumar, S. Sharma and R. Mitra, "Design of Type-2 Fuzzy Controller based on LQR Mapped Fusion Function", Electronics \& Computer Engineering Department, Indian Institute of Technology Roorkee, Uttarakhand, India, (2012).

[8] E. Fresk and G. Nikolakopoulos, "Full quaternion based attitude control for a quadrotor," in Control Conference (ECC), 2013 European, 2013, pp. 3864-3869.

[9] A.S. Vempati, V. Choudhary, L. Behera, "Quadrotor: Design, Control and Vision Based Localization," IFAC Proceedings Volumes 2014, Volume 47, pp. 1104-1110.

[10] G. T. Poyi, "A Novel Approach To The Control Of Quad-Rotor Helicopters Using FuzzyNeural Networks," University of Derby, UK, (2014).

[11] S. Bouabdallah, "Design and control of quadrotors with application to autonomous flying," 2007.

[12] V. S. Gonzalez, V. J. Moreno, "Motion Control of a Quadrotor Aircraft via Singular Perturbations," International Journal of Advanced Robotic Systems 2013, Vol. 10, pp. 368-383.

[13] B. Abci, G. Zheng, D. Efimov, M. Najjar, "Robust Altitude and Attitude Sliding Mode Controllers of Quadrotor," IFAC-Papers OnLine 2017, Volume 50, pp. 2720-2725.

[14] H. Bolandi, M. Rezaei, R. Mohsenipour, H. Nemati, and S.M. Smailzadeh, "Attitude control of a quadrotor with optimized PID controller," Intelligent Control and Automation, 2013, Vol. 4, pp. 335-342.

[15] L. Setlak, R. Kowalik, "Examination of the Unmanned Aerial Vehicle," AMCSE 2018, ITM Web of Conferences, Vol. 24, 2018.

[16] L. Salih, M. Moghavvemi, H.A. Mohamed, and K.S. Gaeid, "Modelling and PID controller design for a quadrotor unmanned air vehicle," in Automation Quality and Testing Robotics (AQTR), 2010 IEEE International Conference, pp. 1-5, 2010.

[17] K.J. Latawiec, R. Stanisławski, W.P. Hunek, Łukaniszyn, M., Laguerre-based modeling of fractional-order LTI SISO systems. In: Proceedings of the 18th International Conference on Methods and Models in Automation and Robotics, Miedzyzdroje, Poland, 2013, pp. 64-69.

[18] K. J. Latawiec, M. Łukaniszyn, and R. Stanisławski (Editors), Advances in Modelling and Control of Non-integer Order Systems, 6th Conference on Non-integer Order Systems Calculus and Its Applications, 2014 Opole, Poland, Lecture Notes in Electrical Engineering, Vol. 320, Springer 2015, pp. 221228.

[19] A. K. Shastry, A. Pattanaik, M. Kothari, "Neuro-adaptive Augmented Dynamic Inversion Controller for Quadrotors," IFAC-Papers On-Line, Vol. 49, 2016, pp. 302-307.

[20] M. Chipofya, D.J. Lee, and K.T. Chong, "Trajectory tracking and stabilization of a quadrotor using model predictive control of Laguerre functions," in Abstract and Applied Analysis, 2015.

[21] B. J. Emran, M. Al-Omari, M. Abdel-Hafez, M.A. F. Jaradat, "A cascaded approach for quadrotor's attitude estimation," Procedia Technology 2014, Volume 15, pp. 268-277.

[22] S. Bouabdallah and R. Siegwart, "Full control of a quadrotor," in Intelligent robots and systems, IROS 2007. IEEE/RSJ international conference on, 2007, pp. 153-158.

[23] A. Rahman, and S. M. Ali, "Design And Analysis Of A Quadratic Optimal Control System for a Type One Plant Model," Department of Electronics and Communication Engineering, National University of Singapore, Singapore; School of Mechanical \& Aerospace Engineering, Nanyang Technological University, Singapore, (2013).

[24] Li J., and Li Y., "Dynamic analysis and PID control for a quadrotor," in Mechatronics and Automation (ICMA), 2011 International Conference on Mechatronics and Automation, 2011, pp. 573-578.

[25] A. Modirrousta, M. Khodabande, "A novel nonlinear hybrid controller design for an uncertain quadrotor with disturbances," Aerospace Science and Technology 2015, Vol. 45, pp. 294-308.

[26] K. Hassani, and W. Lee, "Optimal Tuning of Linear Quadratic Regulators Using Quantum Particle Swarm Optimization," University of Ottawa, School of Electrical Engineering and 
Computer Science, Ottawa, Ontario, Canada, No. 59, 2014, pp. 1-8.

[27] L. Setlak, R. Kowalik, "The dynamics of the movement of formations of unmanned aerial vehicles," WSEAS Transactions on Applied and Theoretical Mechanics, Volume 14, 2019, pp. 192-197.

[28] L. E. Romero, D. F. Pozo, and J. A. Rosales, "Quadcopter stabilization by using PID controllers," Maskana, 2016, pp. 175-186.

[29] K. Ogata, "Modern control engineering, 5th ed.," Boston: Prentice-Hall, (2010).

[30] R. Perez, J. Moreno, R. Miranda, "A Robust Approach for Trajectory Tracking Control of a Quadrotor with Experimental Validation," ISA Transaction 2016, Vol. 65, pp. 262-274.

[31] L. Setlak, R. Kowalik, "Algorithm controlling the autonomous flight of an unmanned aerial vehicle based on the construction of a glider," WSEAS Transactions on Applied and Theoretical Mechanics, Vol. 14, 2019, pp. 5665.

[32] A. Dharan, S. H. O. Storhaug, and H. Karimi, R., "LQG Control of a Semi-active Suspension System equipped with MR rotary brake," Department of Engineering, Faculty of Engineering and Science, University of Agder, N-4898 Grimstad, Norway, (2012).

[33] X. Shao, Q. Meng, J. Liu, H. Wang, "Rise and Disturbance Compensation Based Trajectory Tracking Control for a Quadrotor UAV without Velocity Measurements," Aerospace Science and Technology 2018, Vol. 74, pp. 145-159.

[34] A. E. Bryson, "Control of Spacecraft and Aircraft," Princeton, NJ: Princeton University Press, (2015).

[35] L. Setlak, R. Kowalik, "Stability evaluation of the flight trajectory of unmanned aerial vehicle in the presence of strong wind," WSEAS Transactions on Systems and Control, Vol. 14, 2019, pp. 43-50.

[36] J. Stevek, M. Fikar, "Teaching Aids for Laboratory Experiments with AR. Drone Quadrotor," IFAC274 Papers On-Line 2016, Volume 49, pp. 236-241. 